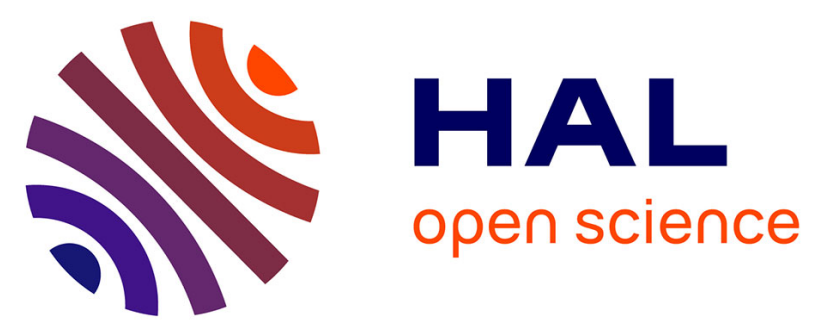

\title{
Interplay between the genetic clades of Micromonas and their viruses in the Western English Channel
}

Anne-Claire Baudoux, H Lebredonchel, H Dehmer, M Latimier, R Edern, Fabienne Rigaut-Jalabert, P Ge, Laure Guillou, Elodie Foulon, Yves Bozec, et al.

\section{To cite this version:}

Anne-Claire Baudoux, H Lebredonchel, H Dehmer, M Latimier, R Edern, et al.. Interplay between the genetic clades of Micromonas and their viruses in the Western English Channel. Environmental Microbiology Reports, 2015, 7 (5), pp.765-773. 10.1111/1758-2229.12309 . hal-01196410

\section{HAL Id: hal-01196410 https://hal.sorbonne-universite.fr/hal-01196410}

Submitted on 9 Sep 2015

HAL is a multi-disciplinary open access archive for the deposit and dissemination of scientific research documents, whether they are published or not. The documents may come from teaching and research institutions in France or abroad, or from public or private research centers.
L'archive ouverte pluridisciplinaire HAL, est destinée au dépôt et à la diffusion de documents scientifiques de niveau recherche, publiés ou non, émanant des établissements d'enseignement et de recherche français ou étrangers, des laboratoires publics ou privés. 
1 Interplay between the genetic clades of Micromonas and their viruses in the Western

2 English Channel

3

4 A-C. Baudoux $^{1,2^{*}}$, H. Lebredonchel ${ }^{3,4}$, H. Dehmer ${ }^{1,2}$, M. Latimier ${ }^{1,2}$, R. Edern ${ }^{1,2}$, F. Rigaut-

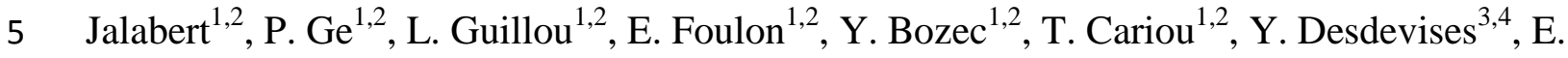

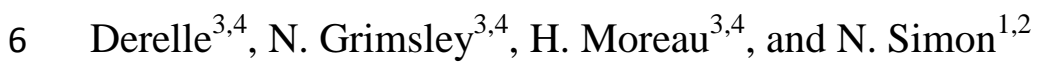

7

$8{ }^{1}$ Sorbonne University, UPMC Paris 06, Station Biologique de Roscoff, UMR7144, Roscoff, 9 France

${ }^{2}$ CNRS, Station Biologique de Roscoff, UMR7144 and FR2424, Roscoff, France

${ }^{3}$ Sorbonne University, UPMC Paris 06, Observatoire Océanologique de Banyuls-sur-mer, UMR7232, Banyuls-sur-mer, France

${ }^{4}$ CNRS, Observatoire Océanologique de Banyuls-sur-mer, UMR7232, Banyuls-sur-mer, France

Running title: Micromonas - virus interactions
7

For correspondence. Email acbaudoux@ @b-roscoff.fr; Tel (+33)2 982925 37; Fax (+33)2 98 292324 


\section{Abstract}

The genus Micromonas comprises distinct genetic clades that commonly dominate eukaryotic phytoplankton community from polar to tropical waters. This phytoplankter is also recurrently infected by abundant and genetically diverse prasinoviruses. Here we report on the interplay between prasinoviruses and Micromonas with regards to the genetic diversity of this host. During one year, we monitored the abundance of 3 clades of Micromonas and their viruses in the Western English Channel both in the environment, using clade-specific probes and flow cytometry, and in the laboratory, using clonal isolates of Micromonas clades to assay for their viruses by plaque-forming units. We showed that the seasonal fluctuations of Micromonas clades were closely mirrored by the abundances of their corresponding viruses, indicating that the members of Micromonas genus are susceptible to viral infection, regardless of their genetic affiliation. The characterization of 45 viral strains revealed that Micromonas clades are attacked by specific virus populations, which exhibit distinctive host clade specificity, life history traits, and genetic diversity. However, some viruses can also cross-infect different host clades suggesting a mechanism of horizontal gene transfer within the genus Micromonas . This study provides novel insights into the impact of viral infection for the ecology and evolution of the prominent phytoplankter Micromonas.

Key words: Mamiellophyceae, Phycodnaviridae, Prasinovirus, host specificities, life history traits, DNA polymerase, English Channel 


\section{Introduction}

Viruses are undoubtedly the most abundant biological entities in the ocean and they intimately interact with every facet of the marine biosphere. Through these interactions, viruses profoundly influence the global biogeochemical cycles by altering the structure and the function of marine communities and by contributing to the cycling of major elements (for review see Suttle, 2005, 2007; Sime-Ngando, 2014). In spite of their global-scale implications, the nature and the dynamics of virus-host interactions in marine environments are far from understood and many basic, yet fundamental, questions remain unsolved: Who are the hosts infected by marine viruses? What are the infection strategies evolved by viruses? How do virus-host interactions vary in time and space? In this respect, characterizing the interplay of ecologically relevant virus-host model systems is a prerequisite for advancing our understanding of virus impacts in nature.

Viruses that infect the cosmopolitan green picoalga Micromonas were first reported by (Pienaar, 1976) and they were found to co-occur with their hosts in a wide range of marine environments, from temperate to cold waters (e.g., Cottrell and Suttle, 1991, 1995a; Sahlsten, 1998; Zingone et al., 1999). The majority of known Micromonas viruses belong to the genus Prasinovirus in the Phycodnaviridae family. Virions are tailless, they exhibit icosahedral capsids of $100-125 \mathrm{~nm}$ in diameter and dsDNA genome of approx. $200 \mathrm{~kb}$ (Mayer and Taylor 1979; Cottrell and Suttle 1991; Zingone et al., 1999). Yet, these prasinoviruses have variable tolerance to chloroform (Martinez et al. 2015) suggesting ultrastructural divergence and they exhibit significant level of genetic variation (Cottrell and Suttle, 1995b). These viruses display a marked seasonal dynamics (Sahlsten, 1998; Zingone et al., 1999) and they were shown to cause considerable amount of mortality in their host population (Cottrell and Suttle, 1995a; Evans et al., 2003). Micromonas strains show a great variability with respect to their susceptibility to infection, indicating that viruses do not only quantitatively but also 
qualitatively regulate their host populations (Sahlsten, 1998; Zingone et al., 1999, 2006). So far, the infection patterns and processes underlying these complex interactions are poorly understood. Of particular interest, the relation between the susceptibility to infection and the genetic diversity of Micromonas has seldom been investigated (Zingone et al., 2006).

The ubiquitous genus Micromonas is genetically diverse and comprises an assemblage of 3 (Guillou et al., 2004) to 5 (Šlapeta et al., 2006; Worden, 2006) discrete phylogenetic clades (or lineages) of flagellated cells that correspond, at least, to 3 distinct species (Simon, unpublished). These clades are often sympatric in marine ecosystems (Foulon et al., 2008; Šlapeta et al., 2006). Yet, their relative contribution to total Micromonas abundance varies in time and space, suggesting that they occupy specific niches (Foulon et al., 2008). The factors that regulate this clade dynamics are not clearly understood. In all likelihood, Micromonas clades exhibit differential responses to abiotic factors but they might also respond differently to predation risks, including those imposed by viruses.

To test whether Micromonas clades display differential sucspetibility to virus infection, we combined field and laboratory experiments on 45 novel viral isolates in order to characterize the interactions between the genetic clades of Micromonas and their viruses in the Western English Channel (WEC) throughout the year 2009. The WEC constitutes an ideal study site where Micromonas is known to dominate the picophytoplankton community (Not et al., 2004). The three main Micromonas genetic types, designated as clades [A.ABC.12], [B.E.3], and [C.D.5] (Worden, 2006) and here referred to as clades A, B, and C, respectively, are recorded year round in this ecosystem (Foulon et al., 2008). Our study revealed that Micromonas clades interact with specific viral populations that display distinctive dynamics and life history traits.

\section{Results and Discussion}


Physico-chemistry at the sampling station

Strong tidal mixing produces a permanently mixed water column at the long term monitoring coastal station SOMLIT-Astan $\left(48^{\circ} 46^{\prime} \mathrm{N}, 3^{\circ} 58^{\prime} \mathrm{W}\right.$, Marrec et al., 2013). During the sampling period (February to December 2009), the water temperature progressively increased from $8.8^{\circ} \mathrm{C}$ in February to a maximum of $16.9^{\circ} \mathrm{C}$ in September while salinity varied between 34.8 (March) and 35.3 (September). Nutrient dynamics varied according to the classical pattern observed in this area (Not et al., 2004). Phosphate and nitrate concentrations showed comparable dynamics with minima recorded in summer $(0.16$ and $0.7 \mu \mathrm{M}$, respectively) and maxima during the winter period $(0.53$ and $11 \mu \mathrm{M}$, respectively). Detailed dynamics of biogeochemical variables at SOMLIT-Astan are publicly available on the observatory website (http://somlit-db.epoc.u-bordeaux1.fr/bdd.php).

\section{Dynamics of Micromonas clades in the Western English Channel}

The phytoplankton community at SOMLIT-Astan during this period was numerically dominated by picophytoplanktonic cells, which comprised, on average, $90 \%$ of the total community in 2009 (data not shown). Among these, the abundance of the picoeukaryotes varied between 2.1 and $16.5 \times 10^{3}$ cells $\mathrm{mL}^{-1}$ with Micromonas spp. accounting for $20 \%$ to $80 \%$ of the total counts as shown by a combination of flow cytometry (FCM) and TSA-FISH analyses (Fig. 1A). As reported previously, this prominent genus displayed marked seasonal dynamics with major peaks of abundance recorded in late spring $\left(6.6 \times 10^{3}\right.$ cells $\left.\mathrm{mL}^{-1}\right)$, early summer $\left(6.3 \times 10^{3}\right.$ cells $\left.\mathrm{mL}^{-1}\right)$, and mid-autumn $\left(4.4 \times 10^{3}\right.$ cells $\left.\mathrm{mL}^{-1}\right)($ Foulon et al., 2008 ; Not et al., 2004).

The 3 main Micromonas genetic clades (hereafter referred to as clades A, B, and C) exhibit recurring seasonal dynamics in the WEC according to previous time series (Foulon et 
al., 2008). We confirmed this pattern of succession in 2009 (Fig. 1B). Micromonas clade A numerically dominated the bloom from February to mid-May comprising 63 to $77 \%$ of the total counts. From June to December, both genetic types A and B equally contributed to Micromonas counts, although clade B cells showed a sudden, yet unexplained, drop in abundance on June 16. Cells that belonged to clade $\mathrm{C}$ were the least abundant accounting, on average, for $7 \pm 6 \%$ of the total counts with somewhat higher contribution during the winter period (14\%) than the remainder of the year $(5 \%)$.

The ecological processes that influence the shifts in the relative abundance of Micromonas clades are unclear. It is traditionally believed that global-scale distribution and diversity of species are mostly driven by ability to withstand abiotic controls (Wiens et al. 2011). Previous study on the global distribution of Micromonas clades suggested that clade B tend to thrive in warmer coastal waters whereas clade $\mathrm{C}$ mostly occur during low-light conditions (Foulon et al., 2008). At local spatial extents, it is however well accepted that species distribution is also influenced by biotic controls (Wiens et al. 2011). It is thus likely that besides eco-physiological adaptations, biotic interactions also regulate the observed Micromonas clade dynamics in the Western English Channel.

\section{Dynamics of Micromonas viruses in the Western English Channel}

Viruses that infect Micromonas (hereafter referred to as MicV), mainly prasinoviruses, have been observed and isolated from different oceanic regions (Cottrell and Suttle, 1991, 1995a; Sahlsten, 1998; Zingone et al., 1999; Short and Short, 2008). FCM analyses of cultured isolates showed that these prasinoviruses consistently cluster within a well-defined population based on their nucleic acid fluorescence (upon Sybr green I staining) and side scatter properties (Fig. S1). In the WEC, this specific population accounted for a small but significant proportion (on average 10\%) of the total virus counts and displayed a marked seasonal dynamics with concentration ranging from $3.4 \times 10^{5} \mathrm{~mL}^{-1}$ in February to $8.8 \times 10^{5}$ 
$143 \mathrm{~mL}^{-1}$ in July 2009 (Fig. 2A). This FCM population probably does not exclusively comprise 144 Micromonas viruses, but counts of this viral population covary strikingly well with 145 Micromonas abundances $(\mathrm{r}=0.675, \mathrm{n}=20, \mathrm{p}<0.005)$, suggesting that viruses might perhaps 146 control abundance of this genus during the sampling period.

To further investigate this hypothesis, we quantified infectious MicV in the WEC by 148 plaque assay using cultures of each 3 Micromonas clades. Significant differences in the number of PFU were detected depending on the phylotype of the host culture (Fig. 2B). As also recorded for their hosts, PFU obtained on Micromonas clade A and B (hereafter, PFU-A and PFU-B, respectively) were substantially more abundant than PFU formed on clade Chosts (hereafter PFU-C). During the sampling period, PFU-A abundance increased concomitantly with the development of Micromonas clade A. Their maximum abundance (8 $\times 10^{2}$ virus $\mathrm{mL}^{-1}$ ) was observed 2 weeks after the peak of host abundance (Figs. 1B and 2B). The monitoring of PFU-B showed low concentration during the winter-spring period but their dynamics was tightly coupled to their host abundance from the summer period until the end of the year, reaching a peak of $11 \times 10^{2}$ virus $\mathrm{mL}^{-1}$ in autumn. Regarding PFU-C, infectious particles were detected year round but their abundance was 10 to 100 -fold lower than PFU-A and -B with no clear relation to their specific host dynamics. Because of variable strain susceptibilities to viral infection, it is difficult to compare our results to former PFU monitoring on Micromonas host cell clades although maximal abundance were within the same range $\left(1.0-4.6 \times 10^{3} \mathrm{PFU} \mathrm{mL}^{-1}\right.$, Cottrell and Suttle, 1991; Sahlsten, 1998; Zingone et al., 1999). For the same reason, this assay most likely underestimates the global abundance of viruses that infect each Micromonas phylotype. Nonetheless, it provides unequivocal evidence that Micromonas, regardless of their genetic clade, were subject to viral attack year round in the WEC. 
The above results led us to question whether specific virus populations are associated to Micromonas clades and, if so, how these virus populations interact with their hosts. To tentatively address this question, 45 viruses (14-16 per host clade) were isolated throughout the sampling period and they were characterized. These viruses were selected randomly from a collection of 176 viral isolates obtained from plaques on Micromonas clade A, B, and C along the year 2009. Regardless of the host on which they were isolated, MicVs exhibited sizeable icosahedral capsids (110 to $130 \mathrm{~nm}$ in diameter) as determined by negative staining using transmission electron microscopy, and large dsDNA genome, as suggested by their flow cytometry signature upon Sybr green I staining (Fig. S1). We therefore assigned our isolates to the virus family Phycodnaviridae and the genus Prasinovirus.

Host specificities - The host range of these 45 prasinoviruses was then determined by pairwise infection using 14 strains belonging to the 3 Micromonas clades (Table 1, Table S1). The large majority of our isolates (35 MicVs) were strictly clade-specific. They infected host strains that belonged only to the clade of their initial host. The remaining 10 viruses could propagate at comparable yield on hosts belonging to clades A and B. Six viruses (out of 15) isolated on a clade A strain could also infect clade B strains, and 4 viruses (out of 14) isolated on a clade B strain could also infect clade A strains. Within each main pattern of specificity (A, B, AB, and $\mathrm{C}$ ), a remarkable clonal diversity was observed with 26 unique specificity profiles that included variable virus infection types ranging from generalist to specialist.

In order to test the structure of this infection network, we applied a network-based analysis according to Flores et al. (2011) and Weitz et al. (2013). As observed previously for phage-bacteria infection networks (Flores et al., 2011), the structure of Micromonas - virus interactions was statistically modular. This statistical analysis applied at the whole matrix 
scale and intra-module scale discriminated 3 modules, which comprised the viruses isolated on hosts from clade A, clade B, and clade C, respectively (Fig. S2). Altogether, these results suggest that Micromonas clade A, B, and C interact with distinct, yet diversified, viral populations. The observed variability in strain-specificity indicates that viruses influence the intraspecific diversity within each Micromonas clades. We also showed that host switches can occur between the more closely related host clades (those that belong to clades A and B), suggesting that viruses could also promote Micromonas diversity through horizontal gene transfers within this genus.

Virus infection strategies - One-step growth experiments conducted on 4 to 5 representative viruses per host clade indicated a similar clade-specific grouping of our isolates. This assay provided estimates of the virus burst size and latent period, which can be related to the virus life strategy. By analogy to the theory of the r- and K-selection (MacArthur, 1967), opportunistic (the most r-selected) viruses are those that exhibit short generation times and high burst sizes whereas less virulent (the most K-selected) viruses are those that induce low mortality in their host population (Suttle, 2007) and they propagate through latent or chronic infection as observed for specific Ostreococcus viruses (Thomas et al., 2011). Based on these parameters, we detected divergent life strategies in Micromonas viruses depending on the host clades that they attack (Fig. 3). The viruses isolated on clade B hosts tended to be the most virulent with short latent period and relatively high progeny production, regardless of their infection range types (i.e. specialist or generalist) whereas the viruses isolated on clade C strains tended to be the least virulent with generally extended latent period (up to $30 \mathrm{~h}$ ) and moderate to high burst size (Fig. 3). Viruses isolated on clade A hosts exhibited an intermediate phenotype with moderate latent period and low burst sizes. 
In contrast to past theories (trade-offs hypothesis, (Poulin, 1998)), generalism (i.e., viruses that propagate on several host clades) was not associated with any compensatory effect on the burst size or on the latent period. The infection kinetics of the selected viral isolates does not correlate to the growth rates of their host (Fig. S3). , The observed differences in infection strategies could be explained by the dynamics of their respective host clade in the studied area. For example, Micromonas clade $\mathrm{C}$ is present year round at low abundance. It is thus tempting to speculate that viruses specific to this host clade had to evolve long latent periods to be maintained within the host population and fairly high progeny production to increase the probability of encounter with their host (Abedon et al., 2001). By contrast, Micromonas clade B displays a marked seasonal dynamics in the WEC with high abundance recorded between mid-June and end-September. We could thus expect that corresponding viruses had to evolve an opportunist lifestyle to efficiently exploit their transient resources.

\section{Phylogenetic relationships between Micromonas viruses}

To investigate the phylogenetic relationships between these viral isolates, we amplified a fragment of the gene encoding their DNA polymerase (polB, Chen and Suttle, 1996, Table S2). This sequence is well conserved among Phycodnaviridae, yet there is sufficient sequence variability to build phylogenetic relationships between viruses from the same genus (Bellec et al., 2009). In spite of their affiliation to the Phycodnaviridae family, the amplification of the polB gene using the AVS1/AVS2 primers failed for 8 of the 45 selected isolates including $\mathrm{B}-, \mathrm{C}$ - and $\mathrm{AB}$-specific viruses. The reason for this failure is unclear. We cannot exclude that the sequence targeted by the degenerated primers might be too divergent. It is also possible that these polymerases carry an intein that makes the PCR product too long to amplify easily under the tested conditions (Clerissi et al., 2013). 
4). The large majority of these haplotypes (24 out of 29) were new, i.e. different from polB sequences in public databases (BLAST analyses, http://www.ncbi.nlm.nih.gov/). In most cases, viral isolates that belonged to the same haplotype exhibited different specificity patterns, suggesting that polB sequencing underestimates the functional diversity of phycodnaviruses as reported previously (Baudoux and Brussaard, 2005; Bellec et al., 2009; Clerissi et al., 2012). In the phylogenetic trees, polB sequences of viruses infecting clade $\mathrm{C}$ hosts formed a well-supported cluster while viruses that infect clade A and clade B hosts distributed into multiple branches suggesting a higher level of genetic diversity. Interestingly, regardless of the host strain they infect, viruses that were highly specific (that infect one single strain among all strains tested) fell apart from other viruses (i.e. RCC4232 and RCC4240, 4242, 4243, Fig. 4 and Table 1). The prasinoviruses that infect Ostreococcus tauri showed a similar phylogenetic pattern and tended to cluster with counterparts that exhibit similar infection range types (Clerissi et al., 2012).

This recurrent correlation between polB phylogeny and the number of hosts infected by a given prasinovirus is somewhat puzzling. The polB gene is indeed used as a neutral marker of evolution in Phycodnaviridae (Chen and Suttle, 1996; Dunigan et al., 2006), that is, this gene is well-conserved among this virus family whereas specificity markers are expected to be more variable genes (Clerissi et al., 2012). Furthermore, virus-host specificity is typically determined during events upstream the virus DNA replication. Several studies reported that the virus attachment on host cell surface determines the susceptibility of marine plankton to infection (e.g., Tarutani et al., 2006; Mizumoto et al., 2007; Stoddard et al., 2007). In other words, the evolution of resistance to viral infection is mostly due to changes in host surface properties that limit viral attachment. Recently, Thomas et al. (2011) evidenced an intriguing mode of resistance in the prasinophyte Ostreococcus tauri. This study 
demonstrated that prasinoviruses could still attach on resistant strains but they did not produce viral progeny. It is thus likely that the resistance mechanism was not due to a change in the host receptor but rather occurred at the DNA entry or DNA replication stage, in which the polB gene product would be involved. Alternatively, viral attachment and internalisation might be a two-stage process as in reoviruses (Reiter et al., 2011). The notion that DNA replication machinery might be involved in host specificity is of course highly speculative but the repeatedly observed link between polB clustering and prasinovirus infectivity range certainly deserves appropriate investigation.

\section{Concluding remarks}

In the Western English Channel, a complex assemblage of viruses is associated to the dominating picophytoplankton species Micromonas spp. Our study revealed, for the first time, that the main Micromonas genetic clades (clade A, clade B, and clade C) are attacked by specific virus populations which displayed distinctive dynamics and life history traits. The apparently high variability in Micromonas virus specificities suggests that viruses maintain a high genetic diversity within each of these clades. However, host switching can occur (particularly for hosts that belong to the clade A and B) suggesting that virus could also influence Micromonas diversity through horizontal gene transfers. The complete genome sequencing of representative viruses and hosts should provide insightful information on the extent of these putative genetic exchanges. It is of course critical to keep in mind that our model-based approach only reflects a partial picture of the actual diversity of virus-host interactions. Still, these approaches provide invaluable information to improve our understanding of the mechanisms of virus-host interactions as well as the evolution and outcomes of these interactions in natural environments. 


\section{Acknowledgments}

The Captain and the crew of R.V. Neomysis are acknowledged for their excellent assistance onboard. We thank the "Service d'Observation en Milieu Littoral (SOMLIT), INSU-CNRS" from Station Biologique de Roscoff for the data on the biogeochemistry and biology at ASTAN. We are grateful to Sophie Lepanse from the Microscopy core facilities, Morgan Perennou from the GENOMER core facilities at the Station Biologique de Roscoff and David Demory for stimulating discussions. We thank the two anonymous reviewers for their constructive comments on a previous version of this manuscript. This research was funded by the ANR funding agency (PICOVIR; grant no. BLAN07-1_200218 and REVIREC; grant no. 12-BSV7-0006-01).

\section{References}

Abedon, S. T., Herschler, T. D., and Stopar, D. (2001). Bacteriophage latent-period evolution as a response to resource availability. Appl Environ Microbiol 67: 4233-4241.

Baudoux, A. C., and Brussaard, C. P. D. (2005). Characterization of different viruses infecting the marine harmful algal bloom species Phaeocystis globosa. Virol 341: 8090.

Bellec, L., Grimsley, N., Moreau, H., and Desdevises, Y. (2009). Phylogenetic analysis of new Prasinoviruses (Phycodnaviridae) that infect the green unicellular algae Ostreococcus, Bathycoccus and Micromonas. Environ Microbiol Reports 1: 114-123

Brussaard, C. P. D. (2004). Optimization of procedures for counting viruses by flow cytometry. Appl Environ Microbiol 70: 1506-1513.

Chen, F., and Suttle, C. A. (1995). Amplification of DNA polymerase gene fragments from viruses infecting microalgae. Appl Environ Microbiol 61: 1274-1278. 
Chen, F., and Suttle, C. A. (1996). Evolutionary relationships among large double-stranded DNA viruses that infect microalgae and other organisms as inferred from DNA polymerase genes. Virol 219: 170-178.

Clerissi, C., Desdevises, Y., and Grimsley, N. (2012). Prasinoviruses of the marine green alga Ostreococcus tauri are mainly species specific. J Virol 86: 4611-4619.

Clerissi, C., Grimsley, N., and Desdevises, Y. (2013). Genetic exchanges of inteins between prasinoviruses (phycodnaviridae). Evol 67: 18-33.

Cottrell, M. T., and Suttle, C. A. (1991). Wide-spread occurrence and clonal variation in viruses which cause lysis of a cosmopolitan, eukaryotic marine phytoplankter, Micromonas pusilla. Mar Ecol Prog Ser 78: 1-9.

Cottrell, M. T., and Suttle, C. A. (1995a). Dynamics of a lytic virus infecting the photosynthetic marine picoflagellate Micromonas pusilla. Limnol and Oceanogr 40: 730-739.

Cottrell, M. T., and Suttle, C. A. (1995b). Genetic diversity of algal viruses which lyse the photosynthetic picoflagellate Micromonas pusilla (Prasinophyceae). Appl Environ Microbiol 61: 3088-3091.

Dunigan, D. D., Fitzgerald, L. A., and Van Etten, J. L. (2006). Phycodnaviruses: A peek at genetic diversity. Virus Res 117: 119-132.

Edgar, R. C. (2004). MUSCLE: multiple sequence alignment with high accuracy and high throughput. Nucleic Acids Res 32: 1792-1797.

Evans, C., Archer, S. D., Jacquet, S., and Wilson, W. H. (2003). Direct estimates of the contribution of viral lysis and microzooplankton grazing to the decline of a Micromonas spp. population. Aquat Microbial Ecol 30: 207-219.

Flores, C. O., Meyer, J. R., Valverde, S., Farr, L., and Weitz, J. S. (2011). Statistical structure of host-phage interactions. Proc Natl Acad Sci USA 108: E288-E297. 
Foulon, E., Not, F., Jalabert, F., Cariou, T., Massana, R., and Simon, N. (2008). Ecological niche partitioning in the picoplanktonic green alga Micromonas pusilla: evidence from environmental surveys using phylogenetic probes. Environ Microbiol 10 : 2433-2443.

Guillou, L., Eikrem, W., Chrétiennot-Dinet, M.-J., Le Gall, F., Massana, R., Romari, K., et al. (2004). Diversity of picoplanktonic prasinophytes assessed by direct nuclear SSU rDNA sequencing of environmental samples and novel isolates retrieved from oceanic and coastal marine ecosystems. Protist 155: 193-214.

MacArthur, R. H. (1967). The theory of island biogeography (Vol. 1): Princeton University Press.

Marrec, P., Cariou, T., Collin, E., Durand, A., Latimier, M., Macé, E., et al. (2013). Seasonal and latitudinal variability of the CO 2 system in the western English Channel based on Voluntary Observing Ship (VOS) measurements. Mar Chem 155: 29-41.

Martinez Martinez, J., Boere, A., Gilg, I., van Lent, J. W. M.,Witte, H. J., van Bleijswijk J. D. L., Brussaard, C.P.D. (2015) New lipid envelope-containing dsDNA virus isolates infecting Micromonas pusilla reveal a separate phylogenetic group. Aquat Microb Ecol 74: 17-28

Mizumoto, H., Tomaru, Y., Takao, Y., Shirai, Y., and Nagasaki, K. (2007). Intraspecies host specificity of a single-stranded RNA virus infecting a marine photosynthetic protist is determined at the early steps of infection. J Virol 81: 1372-1378.

Not, F., Latasa, M., Marie, D., Cariou, T., Vaulot, D., and Simon, N. (2004). A single species, Micromonas pusilla (Prasinophyceae), dominates the eukaryotic picoplankton in the western English channel. Appl Environ Microbiol 70: 4064-4072. 
Posada, D., and Crandall, K.A. (1998). MODELTEST: testing the model of DNA substitution. Bioinformatics 14: 817-818.

Pienaar, R. N. (1976). Virus-like particles in three species of phytoplankton from San Juan Island, Washington. Phycologia 15: 185-190.

Poulin, R. (1998). Large-scale patterns of host use by parasites of freshwater fishes. Ecol Letters 1: 118-128.

Reiter, D. M., Frierson, J. M., Halvorson, E. E., Kobayashi, T., Dermody, T. S., and Stehle, T. (2011). Crystal structure of reovirus attachment protein $\sigma 1$ in complex with sialylated oligosaccharides. PLoS pathogens 7: e1002166.

Ronquist, F., Teslenko, M., van der Mark, P., Ayres, D. L., Darling, A., Höhna, S., et al. (2012). MrBayes 3.2: efficient Bayesian phylogenetic inference and model choice across a large model space. Syst Biol 61: 539-542.

Sahlsten, E. (1998). Seasonal abundance in Skagerrak-Kattegat coastal waters and host specificity of viruses infecting the marine photosynthetic flagellate Micromonas pusilla. Aquat Microbial Ecol 16: 103-108.

Short, S. M., and Short, C. M. (2008). Diversity of algal viruses in various North American freshwater environments Aquat Microbial Ecol 51: 13-21.

Sime-Ngando, T. (2014). Environmental bacteriophages: viruses of microbes in aquatic ecosystems. Front Microbiol 5: 355

Šlapeta, J., López-García, P., and Moreira, D. (2006). Global dispersal and ancient cryptic species in the smallest marine eukaryotes. Mol Biol Evol 23: 23-29.

Stoddard, L. I., Martiny, J. B., and Marston, M. F. (2007). Selection and characterization of cyanophage resistance in marine Synechococcus strains. Appl Environ Microbiol 73: $5516-5522$.

Suttle, C. A. (2005). Viruses in the sea. Nature 437: 356-361. 
Suttle, C. A. (2007). Marine viruses - major players in the global ecosystem. Nature Rev Microbiol 5: 801-812.

Swofford, D. L. (2003). PAUP: Phylogenetic analysis using parsimony. Version 4. Sunderland, Massachusetts: Sinauer Associates.

Tarutani, K., Nagasaki, K., and Yamaguchi, M. (2006). Virus adsorption process determines virus susceptibility in Heterosigma akashiwo (Raphidophyceae). Aquat Microbial Ecol 42: 209-213.

Thomas, R., Grimsley, N., Escande, M. 1., Subirana, L., Derelle, E., and Moreau, H. (2011). Acquisition and maintenance of resistance to viruses in eukaryotic phytoplankton populations. Environ Microbiol 13: 1412-1420.

Weitz, J. S., Poisot, T., Meyer, J. R., Flores, C. O., Valverde, S., Sullivan, M. B., et al. (2013). Phage-bacteria infection networks. Trends Microbiol 21: 82-91.

Worden, A. Z. (2006). Picoeukaryote diversity in coastal waters of the Pacific Ocean. Aquat Microbial Ecol 43: 165-175.

Zingone, A., Sarno, D., and Forlani, G. (1999). Seasonal dynamics in the abundance of Micromonas pusilla (Prasinophyceae) and its viruses in the Gulf of Naples (Mediterranean Sea). J Plank Res 21: 2143-2159.

Zingone, A., Natale, F., Biffali, E., Borra, M., Forlani, G., and Sarno, D. (2006) Diversity in morphology, infectivity, molecular characteristics and induced host resistance between two viruses infecting Micromonas pusilla. Aquat Microbial Ecol 45: 1-14. 